\title{
Unmasking the ultrahigh-energy cosmic ray origin
}

\author{
Luis A. Anchordoqui* \\ Department of Physics and Astronomy, Lehman College at CUNY, NY 10468, USA \\ Department of Physics, Graduate Center, City University of New York, NY 10016, USA \\ Department of Astrophysics, American Museum of Natural History, NY 10024, USA \\ E-mail: luis.anchordoqui@gmail.com
}

\begin{abstract}
The sharp change in slope of the ultrahigh-energy cosmic ray spectrum around $10^{9.6} \mathrm{GeV}$ (the ankle), combined with evidence of a light but extragalactic component near and below the ankle which evolves to intermediate/heavy composition above, has proved exceedingly challenging to understand theoretically. Recently, we introduced a very general model in which, for a range of source conditions, photo-disintegration of ultrahigh-energy nuclei in the region surrounding the accelerator naturally accounts for the observed spectrum and composition of the entire extragalactic component, which dominates above about $10^{8.5} \mathrm{GeV}$. In this communication we review the generalities of the model and show that starburst galaxies provide a compelling source example.
\end{abstract}

The European Physical Society Conference on High Energy Physics

5-12 July

Venice, Italy

${ }^{*}$ Speaker. 
The ultrahigh-energy $\left(E>10^{9} \mathrm{GeV}\right)$ cosmic ray (UHECR) spectrum can be roughly described by a twice-broken power law [1]. The first break, at an energy $E \sim 10^{9.6} \mathrm{GeV}$, is a hardening of the spectrum known as the ankle, while the second abrupt softening of the spectrum is the UV cutoff at $E \sim 10^{10.6} \mathrm{GeV}$. Optical observations of air showers with fluorescence telescopes or non-imaging Cherenkov detectors consistently find a predominantly light composition at around $10^{9} \mathrm{GeV}$ [2] and the contribution of protons to the overall cosmic ray flux is $\gtrsim 50 \%$ in this energy range [3]. Due to the absence of a large anisotropy in the arrival direction of cosmic rays below the ankle [4], we can conclude that these protons must be of extragalactic origin. At energies above $10^{10} \mathrm{GeV}$, the high-statistics data from the Pierre Auger Observatory suggests a gradual increase of the fraction of heavy nuclei in the cosmic ray flux [3]. Within uncertainties, the data from the Telescope Array (TA) is consistent with these findings [5]. In addition, TA has observed a statistically significant excess in cosmic rays with energies above $10^{10.7} \mathrm{GeV}$ in a region of the sky spanning about $20^{\circ}$, centered on equatorial coordinates $\left(\right.$ R.A. $=146.7^{\circ}$, Dec. $=43.2^{\circ}$ ) [6]. This is colloquially referred to as the TA hot spot. The absence of a concentration of nearby sources in this region of the sky corroborates other experimental evidence for heavy nuclei, whereby a few local sources within our cosmic backyard can produce the hot spot through deflection in the extragalactic and Galactic magnetic fields.

Simultaneously reproducing the Auger spectrum above the ankle together with the observed nuclear composition requires hard source spectra [7]. It is possible to accommodate the entire UHECR data with the addition of an ad hoc light extragalactic component below the ankle, with a steep injection spectrum [8]. However, a more natural explanation of the entire spectrum and composition emerges while accounting for the post-processing of UHECRs through photo-disintegration in the environment surrounding the source [9]. In this model relativistic nuclei accelerated by a central engine to extremely high energies remain trapped in the turbulent magnetic field of the source environment. Their escape time decreases faster than the interaction time with increasing energy, so that only the highest energy nuclei can escape the source unscathed. In effect, the source environment acts as a high-pass filter on the spectrum of cosmic rays. All nuclei below the energy filter interact, scattering off the far-infrared photons in the source environment. These photonuclear interactions produce a steep spectrum of secondary nucleons, which is overtaken by the harder spectrum of the surviving nucleus fragments above about $10^{9.6} \mathrm{GeV}$. These overlapping spectra could then carve an ankle-like feature into the source emission spectrum. The spectrum above the ankle exhibits a progressive transition to heavy nuclei, as the escape of non-interacting nuclei becomes efficient. The model is essentially characterized by two parameters of the source environment: (i) a low gas density and (ii) a thermal background of far- and mid-infrared photons. In addition, reproducing the data with such a model requires a central engine with a hard spectrum $\propto E^{-1}$, as expected for acceleration in young neutron stars [11]. Note that we can distinguish two components contributing to the nucleons populating the spectrum below the ankle: those emitted by the source $\mathscr{N}_{s}$ and those produced during propagation $\mathscr{N}_{p}$.

For a central engine emitting a single nuclear species, the composition that best fit the Auger measurements between the ankle and the cutoff is ${ }^{28} \mathrm{Si}$; see Figs. 1 and 6 in [9]. This is because the energy per nucleon $E_{N}=E / A$ just above the cutoff $E \sim 10^{10.8} \mathrm{GeV}$ has to be roughly the energy just below the ankle $\sim 10^{9.2} \mathrm{GeV}$. Such an energy correlation requires a baryon number $A \sim 30$. The model is also consistent with a composition that follows a Galactic mixture with 10 elements; 
see Fig. 9 in [9]. For the case of TA data, the favor composition is heavier and partially consistent with pure iron; see Fig. 8 in [9].

The possibilities initially entertained conceptually may be brought to realization in galaxies undergoing periods of rapid star formation, the so-called "starbursts." These environments feature strong infrared emission by dust associated with high levels of interstellar extinction, strong UV spectra from the Lyman $\alpha$ emission of hot OB stars, and considerable radio emission produced by recent supernova remnants. The central regions of starburst galaxies can be orders of magnitude brighter than those of normal spiral galaxies. By now it is well-established that from the central active region a galactic-scale superwind is driven by the collective effect of supernovae and winds from massive stars [10]. The high supernovae rate creates a cavity of hot gas $\left(\sim 10^{8} \mathrm{~K}\right)$ whose cooling time is much greater than the expansion time scale. In other words, the wind has a sufficiently low density that it will move out before cooling. Since the wind is sufficiently powerful, it can blow out of the interstellar medium of the galaxy as a hot bubble. As the cavity expands a strong shock front is formed on the contact surface with the cool interstellar medium. Observations of local starburst superwinds seem to indicate that they are a complex multiphase phenomena. When the cooler material of interstellar clouds encounters the wind, it is heated and accelerated, giving rise to regions of optical line emission. The dust contained in these clouds is revealed as it reddens the background starlight and scatters the starburst's UV radiation. Warm (100 $\lesssim T / \mathrm{K} \lesssim 200)$ dust in the outer layers of the clouds radiates substantially at mid-infrared wavelength $(530 \lesssim \lambda / \mu \mathrm{m} \lesssim 300)$, and the cooler dust heated to $30 \lesssim T / \mathrm{K} \lesssim 60$ radiates in the far-infrared range $(30 \lesssim \lambda / \mu \mathrm{m} \lesssim 300)$. All in all, the superwind environment satisfies the two essential requirements advocated in [9]. Moreover, because of the high prevalence of supernovae, starbursts must possess a large density of newly-born neutron stars. As noted above, neutrons stars (with their metal-rich surfaces) are potentially candidate sources for the central engine: iron nuclei can be stripped off the surface and be accelerated through unipolar induction up to the maximum observed energies, and with the appropriate hard spectrum $\propto E^{-1}$ [11]. For surface temperatures exceeding $10^{6} \mathrm{~K}$, photo-disintegration in the thermal radiation fields generated by the star starts to be effective and with increasing temperature a large fraction of the initial iron nuclei is transformed into a mixed composition of lighter nuclei [12], akin to that shown in Fig. 9 of [9].

An interesting twist is that after the nuclei escape from the central region of the galaxy and they are injected into the galactic-scale superwind they could potentially experience further acceleration at its terminal shock. The superwind environment could play the role of a high pass filter, but at the same time could allow re-acceleration of primary and secondary particles. The shock velocity $v_{\mathrm{sh}}$ can be estimated from the empirically determined superwind kinetic energy flux $\dot{E}_{\mathrm{sw}}$ and the mass flux $\dot{M}$ generated by the starburst through $\dot{E}_{\mathrm{sw}}=\dot{M} v_{\mathrm{sh}}^{2} / 2$. The shock radius can be approximated by $r_{\mathrm{sh}} \approx v_{\mathrm{sh}} \tau$, where $\tau$ is the starburst age. Since the age is about a few tens to hundreds of million years, the maximum energy attainable in this configuration is constrained by the limited acceleration time provided by the shock's finite lifetime. In terms of parameters that can be determined from observations, for a nucleus of charge $Z e$, we have $E \sim Z e \bar{B} \tau \dot{E}_{\mathrm{sw}} /(2 \dot{M})$, where $\bar{B}$ is the average magnetic field [13]. The predicted kinetic energy and mass fluxes of starbursts, derived from the measured IR luminosity, are $2 \times 10^{42} \mathrm{erg} \mathrm{s}^{-1}$ and $1.2 \mathrm{M}_{\odot} \mathrm{yr}^{-1}$, respectively [14]. The starburst age is estimated from numerical models that use theoretical evolutionary tracks for individual stars and make sums over the entire stellar population at each time in order to produce 
the galaxy luminosity as a function of time [15]. Fitting the observational data these models provide a range of suitable ages for the starburst phase, $50 \lesssim \tau / \mathrm{Myr} \lesssim 200$. It has been noted that the magnetic energy density of the starburst region could be significantly higher than that expected from equipartition arguments with comparable cosmic rays and magnetic energy densities [16]. Taking $\tau=50 \mathrm{Myr}$ and $\bar{B} \sim 50 \mu \mathrm{G}$, we obtain $E_{N}^{\max } \sim 10^{9.5} \mathrm{GeV}$. The source emission spectrum would remain hard provided its shape is driven by UHECR nucleus leakage from the boundaries of the shock (a.k.a direct escape) [17].

In closing we note that the more involved model, which allows for re-acceleration of the photodisintegrated nuclei and the secondary nucleons, could relax the constraint on nuclear composition by potentially increasing the $\mathscr{N}_{s} / \mathscr{N}_{p}$ ratio. Note the difference between trapped re-accelerated particles and those that have escaped the source. Future data from AugerPrime and NASA's Probe Of Multi-Messenger Astrophysics (POEMMA) will provide a unique opportunity to test the model.

I acknowledge many useful discussions with my colleagues of the Pierre Auger Collaboration. LAA is supported by U.S. NSF (Grant No. PHY-1620661) and NASA (Grant No. NNX13AH52G).

\section{References}

[1] R. U. Abbasi et al. [HiRes Collaboration], Phys. Rev. Lett. 100, 101101 (2008); J. Abraham et al. [Pierre Auger Collaboration], Phys. Rev. Lett. 101, 061101 (2008); Phys. Lett. B 685, 239 (2010).

[2] K. H. Kampert and M. Unger, Astropart. Phys. 35, 660 (2012).

[3] A. Aab et al. [Pierre Auger Collaboration], Phys. Rev. D 90, 122005 (2014); Phys. Rev. D 90,122006 (2014); Phys. Lett. B 762, 288 (2016); JCAP 1704, 038 (2017).

[4] P. Abreu et al. [Pierre Auger Collaboration], Astrophys. J. Suppl. 203, 34 (2012); A. Aab et al. [Pierre Auger Collaboration], Astrophys. J. 802, 111 (2015).

[5] R. U. Abbasi et al., Astropart. Phys. 64, 49 (2014); R. Abbasi et al. [Pierre Auger and Telescope Array Collaborations], JPS Conf. Proc. 9, 010016 (2016).

[6] R. U. Abbasi et al. [Telescope Array Collaboration], Astrophys. J. 790, L21 (2014).

[7] A. Aab et al. [Pierre Auger Collaboration], JCAP 1704, 038 (2017).

[8] R. Aloisio, V. Berezinsky and P. Blasi, JCAP 1410, 020 (2014).

[9] M. Unger, G. R. Farrar and L. A. Anchordoqui, Phys. Rev. D 92, 123001 (2015).

[10] S. Veilleux, G. Cecil and J. Bland-Hawthorn, Ann. Rev. Astron. Astrophys. 43, 769 (2005)

[11] P. Blasi, R. I. Epstein and A. V. Olinto, Astrophys. J. 533, L123 (2000); K. Fang, K. Kotera and A. V. Olinto, Astrophys. J. 750, 118 (2012); JCAP 1303, 010 (2013).

[12] K. Kotera, E. Amato and P. Blasi, JCAP 1508, no. 08, 026 (2015).

[13] L. A. Anchordoqui, G. E. Romero and J. A. Combi, Phys. Rev. D 60, 103001 (1999);

D. F. Torres and L. A. Anchordoqui, Rept. Prog. Phys. 67, 1663 (2004);

L. A. Anchordoqui, V. Barger and T. J. Weiler, arXiv:1707.05408.

[14] T. M. Heckman, L. Armus and G. K. Miley, Astrophys. J. Suppl. 74, 833 (1990).

[15] G. H. Rieke, M. J. Lebofsky, R. Thompson, F. Low and A. Tokunaga, Astrophys. J. 238, 24 (1980).

[16] T. A. Thompson, E. Quataert, E. Waxman, N. Murray and C. Martin, Astrophys. J. 645, 186 (2006).

[17] P. Baerwald, M. Bustamante and W. Winter, Astrophys. J. 768, 186 (2013). 